\title{
Oggi siamo tutti dantofili: è il primo Dantedì della storia
}

\section{Claudio Marazzini}

PUBBLICATO: 25 MARZO 2020

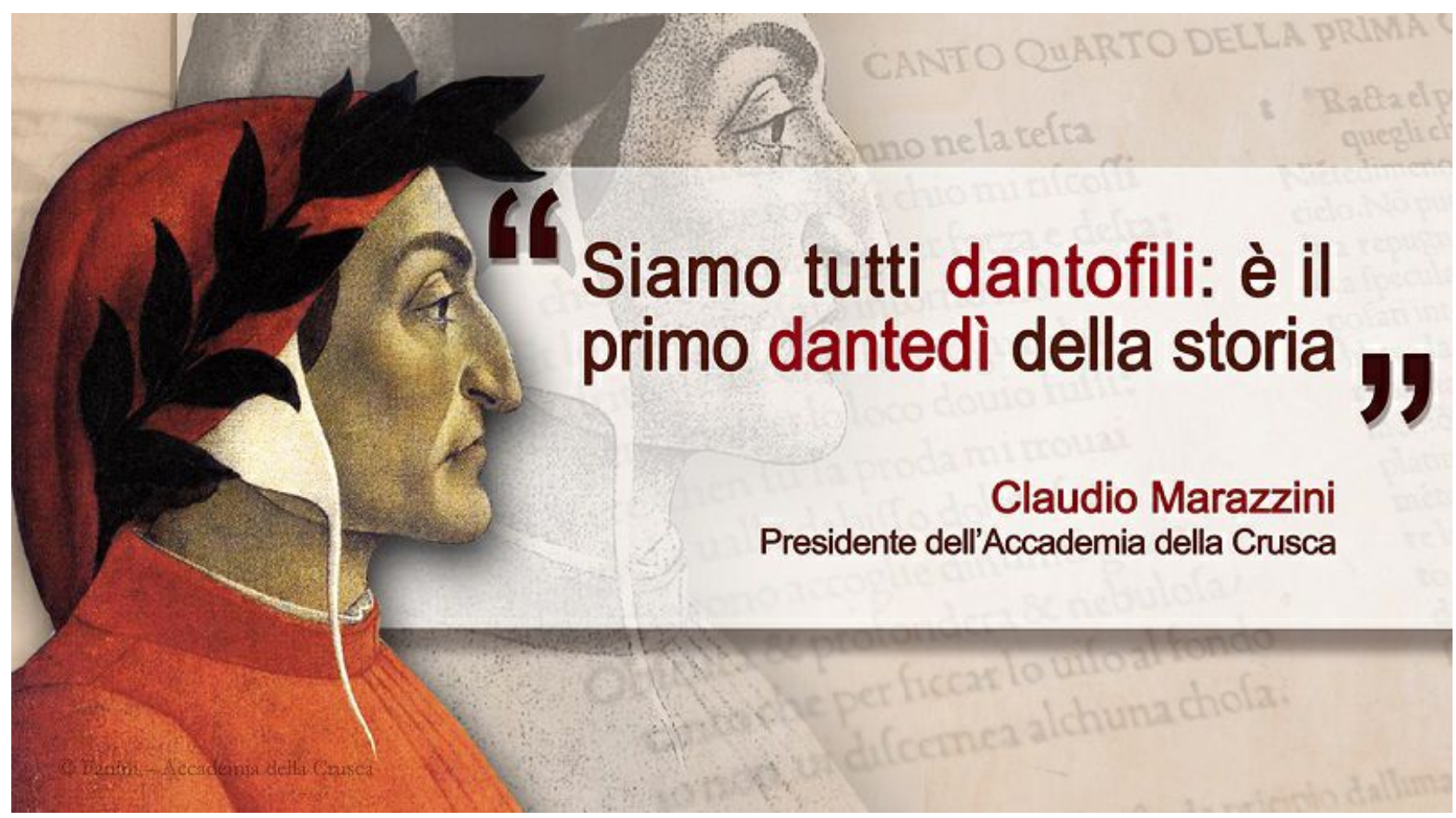

Il primo Dantedi della storia

Oggi si celebra il primo "Dantedi" della storia. È caduto in un periodo sfortunato. Il programma, fino a un mese fa, era ambizioso: volevamo coinvolgere nelliniziativa la scuola, mobilitando studenti e professori, radunandoli in cinema e teatri, o addirittura sfilando per strada, come al tempo delle celebrazioni dantesche fiorentine del i865. Fantasticavamo sul coinvolgimento di un gran numero di persone. Non sarà così. L'esercizio sarà tutto virtuale.

A questo si è preparata anche la Crusca, come il MIBACT, il nostro Ministero dei beni culturali. Lanciamo oggi, attraverso i nostri canali social, una serie di interviste e di filmati in cui si parla di Dante. Giova inoltre ricordare che stiamo lavorando per il 202I, quando le celebrazioni dantesche avranno un rilievo speciale, a 700 anni dalla morte del Poeta. In vista di quella data, la Crusca ha messo in cantiere, assieme all'OVI-CNR, il Vocabolario dantesco volgare, e allo stesso tempo è stato avviato, con collaborazione ancora più ampia, il Vocabolario dantesco latino.

Il Dantedi non prevedeva celebrazioni troppo accademiche o specialistiche, ma attività divertenti, persino spettacolari. Celebrato oggi, nelle condizioni di mobilità ridotta al minimo o a zero, nello stato di necessità che costringe la gente a cantare dai balconi e organizzare flash-mob sospesi (prima, infatti, il flash-mob aveva un senso diverso: trattandosi, come dice l'Oxford dictionary, di "a large public gathering at which people perform an unusual or seemingly random act and then disperse", i luoghi deputati erano le piazze, non i balconi, che tuttavia ora ben si adattano a una rappresentazione dei regni danteschi dell'aldilà). 
Tuttavia il Dantedì è un'occasione per il nostro orgoglio nazionale, quello che fa ripetere a molti il "ce la faremo", se non altro di buon auspicio. Si sventolano bandiere, da quei balconi, e si recitano versi danteschi, come ha suggerito Francesco Sabatini. Insomma, oggi siamo tutti dantofili.

\section{Dantofilo: storia di una parola}

Poiché la Crusca si occupa di parole, proveremo a dire qualche cosa proprio sulla parola dantofilo, che è cosa diversa dal dantista. Il dantista è l'esperto dell'opera di Dante, come i colleghi dantisti accademici della Crusca; il dantofilo è chi "coltiva lo studio e la lettura di Dante" (così il GRADIT), dunque un amatore un po' dilettante, più o meno esperto, non professionista. Molto accurata la definizione del dizionario Treccani di A. Duro: "composto del nome di Dante e -filo. - Chi, o che, studia assiduamente Dante; ma soprattutto chi ne raccoglie edizioni, traduzioni, ecc., o fa di lui un culto, anche senza serî propositi scientifici". Il dantofilo è uno che ama Dante, come oggi tutti lo amiamo in questo primo Dantedi. Il GRADIT, il Grande dizionario italiano dell'uso, che registra Dantofilo nel vol. II, p. 466, mette accanto a Dantofilo una data: 1956. È il gioco dell'anagrafe delle parole, a cui si dedica anche la Crusca, che ha allestito la banca dati ArchiDATA, ideata e diretta da Ludovica Maconi: si tratta della grande banca in cui si aggiornano le date delle parole italiane, cioè si individua la loro prima attestazione, da cui ha inizio la loro storia.

Ebbene, la data 1956 del GRADIT per dantofilo va proprio corretta. Per la verità è stata in parte rivista nell'edizione elettronica su chiavetta: lì la data è r $879^{2}$. Questa seconda data è già meglio dell'altra (la quale è frutto di un errore marchiano, perché deriva da una cattiva interpretazione di un giusto rinvio del GDLI "Battaglia"3). Tuttavia i "dantofili" c'erano già prima del 1956 e anche prima del I879.

\section{Dantofili di tutte le nazioni}

La storia di dantofilo è molto istruttiva. Ci riporta a un momento magico dell'interesse per Dante, e anche ci rammenta la dimensione internazionale degli studi sul nostro maggior poeta.

Siamo in Inghilterra, alla fine dell'Ottocento. Un inglese che sta a Oxford, Edward Moore, studia Dante in maniera formidabile. Nel 1883 presenta le sue ricerche ai membri della "Oxford Dante Society". L'Ottocento è un secolo fondamentale nella storia della filologia. Nel i850 era uscito il Lucrezio di Lachmann. Si stanno riscoprendo i codici come fonte primaria per ricostruire la forma autentica dei testi, eliminando gli errori introdotti nel tempo dai copisti. Si sta imparando a utilizzare i manoscritti antichi in maniera scientifica, con metodo rigoroso, non cavandone le lezioni qua e là secondo scelte soggettive.

Di Dante, come degli autori classici greci e latini, non abbiamo gli autografi. In questo senso, Dante resta misterioso come gli autori più antichi, anche se la distanza storica tra l'originale e i manoscritti giunti a noi non si misura in secoli (come per i classici greco-latini) ma in decenni: il più antico manoscritto della Commedia è del I336, il Landiano della Biblioteca comunale di Piacenza, e dunque non è troppo distante dalla morte di Dante; "si tratta però di anni intensi che segnano in modo indelebile la trasmissione dell'opera" come scrive Alfredo Stussi. Questo vuol dire che già in poco tempo i manoscritti che riproducevano la Commedia si erano riempiti di errori.

La filologia dantesca, nel Cinquecento, passa in maniera altrettanto decisiva attraverso Pietro Bembo, il grande regolatore cinquecentesco dell'italiano. La tradizione della Commedia di Dante, per centinaia di anni, è stata in sostanza affidata a una vulgata che si fondava, pur con ritocchi di vario genere (spesso peggiorativi), sulla famosa aldina del $\mathrm{I}_{502}$, la stampa veneziana della Commedia procurata da 
Bembo per Aldo Manuzio, quella che non s'intitolava nemmeno Commedia o Comedia (e lasciamo perdere il "divina", che è posticcio), ma Le terze rime (con riferimento alla forma metrica, perché la Commedia è in terzine). Bembo si era basato su di un codice appartenente a suo padre, l'odierno Vaticano lat. 3199. Roba di famiglia, dunque: perché il padre di Bembo, Bernardo, a sua volta, da uomo assai colto e intelligente qual era, aveva alimentato il culto di Dante, tanto che aveva provveduto al restauro della tomba del poeta, nel periodo in cui era stato podestà a Ravenna. Anche oggi le lapidi di marmo sulle pareti laterali della tomba ravennate ricordano quel lodevole restauro.

\section{Arriva la filologia}

Nel secolo XIX si comprese finalmente che era necessario interrogare a fondo i manoscritti antichi per rifondare la filologia dantesca. Quali scegliere, però? Non esiste un autografo della Commedia. Non abbiamo nemmeno una pagina o una riga scritta di pugno da Dante. I codici che trasmettono la Commedia sono molti, oltre 6oo. Impossibile allora (e anche oggi non si è risolto il problema) ordinarli in uno "stemma" secondo il metodo di Lachmann. Però, finalmente, nel secolo XIX, si capi che i codici antichi contenevano il testo più affidabile a cui ricorrere, anche se quegli stessi codici non mancavano di errori. La Commedia non ha una tradizione testuale profondamente diversa nei vari manoscritti, e ciò ci rassicura; ma le differenze minute sono moltissime, una miriade, e tali da modificare il significato di molti versi. Gli studiosi dell'Ottocento, dunque affrontarono questo problema.

Non solo gli italiani studiavano Dante. Nel I862, Karl Witte, professore di diritto a Halle, aveva dato un'edizione della Commedia basata su quattro manoscritti, selezionati tra quelli che riteneva molto affidabili. Il saggio con cui Witte presenta le proprie ricerche è ancora oggi un esempio formidabile di rigore e di metodo. Tuttavia quattro manoscritti, scelti un po' a caso tra tanti altri, non potevano bastare. L'oxoniense Moore prese le mosse proprio dal lavoro di Witte, su cui espresse un giudizio positivo, pur rilevandone i limiti. Si trattava però di andare oltre a Witte, superandone i difetti. Non solo Moore stampò la Commedia, ma anche tutte le altre opere di Dante. Nel presentare i propri studi, e nel commentare quelli degli altri dantisti, Moore adoperò più volte la parola Dantofilo, sempre maiuscola (siamo nel I894), come si vede in questa pagina, in cui spiega le sue scelte relativamente al testo della Vita nuova, del De vulgari eloquentia, delle liriche, e soprattutto della Divina Commedia: 
vi

PROEMIO

Inquanto alla condizione dei vari testi delle diverse opere di Dante, a da giudicursi in modo molto differente. Alcune sono stato gia suffeientemente lavorato per la cura e le ricerche altrui. Abbiamo dunque ripetuto quasi letteralmente il testo Wittiano della Vita Nuova e della De Monarchia. Per il permesso di servirci di questi due testi e ristamparli quasi tal quale, i distinti ringraziamenti dei Direttori e dell' Editore sono dovuti alla cortesia dei Signori Brockhaus di Lipsia, e Braumulller di Vienna. Similmento le Epistolae, la Quaestio, ed i Poemi Minori sono stati riprodotti con piecolissime variazioni sul testo del Fraticelli; od anche della licenza conceduta loro a questo fine dai Signori Barbèra e Cin di Firenze essi sono obligatissimi.

Nel De Vulgari Eloquio, adottando genoralmento il testo del Fraticelli, l' abbiamo corretio coll' aiuto del Codice di Grenoble, recentamente riprodotto in facsimile dal Dr. Prompt, tenendo conto inoltre delle sue note critiche, che si trovano nel Proemio di quest' opora. Anche la punteggiatura assai confusa del Fraticelli è stata riveduta.

$\mathrm{L}$ ' indice ò stato compilato dal Signor Paget Toynbee ; e di questo corollario utilissimo all' edizione sono molto tenuti i Direttori a questo egregio - benemerito Dantofilo, siceome di certo lo sarà anche ciascun lettore.

Resta a diro del Canzoniere, della Divinu Comnedia, o del Convito.

II testo del Canzonicre e virtualmente quello del Fraticelli, ma questo ò stato ritoccato, od anche la disposizione delle composizioni riordinata, dal valentes Dantofle Signor York Powell.

Il testo della Divina Commelia abbiamo fondato-e chi potrebbe farne altrimenti, se non volesse 'far ritroso calle' $\varphi$ - su quello del Witte, Berlino, 1862. Ma l' illustre editore di quel testo sarebbe stato il $\cdots$ imo a confessare di non potersi vantare di completezza su questo lavoro in in stante ch' esso, sia un avanzamento importantissimo sopra tutte le $e^{i}$ ar inferiori. Lo stesso Witte dice nei wuoi Prolegumení (p. lxxx), 'Quattró un 'mano l' unico fondamento della presente edizione. Non vi è parola, nöa sillaba, che non si appoggi sull' autorità di almeno uno di quei testi.' E sogginngo un po' di sotto che alcune (benche rarissime) volte $\mathrm{ki}$ sia attenuto ad una lezione di questi manoseritti quantunque non fusse quella che esso. credeva certamento da preferirsi. Ma non si devon ehiudero gli occhi ull immenso spoglio di varianti che la diligenza di parocchi Dantofili ha tratto da diversi codici in questi ultimi anni. Fra altre mi son servito dappertutto delle varie lezioni gia pubblicate nel mio lavoro sul Textual Criticism of the Divina Cummetia, e di moltissime altre da me aocumulato da qualche anno dopo la pubblicazione di quello. Nè sarebbe in alcun modo derogare ull' eccellente testo del Witte, fondato sul principio insufficiente sopradetto, se dopo le ricerche 'fatte da tanti e tanti studiosi per una trentina d' anni o stato necessario d' introdurvi un numero considerevole di emendazioni.

Ma è sopra il testo del Convito che la più grave difficoltà e sopravvenuta, ud in ouesta narte il testo aui strmmato si trova intaramenta riveduto

Benché il libro Tutte le opere di Dante sia pubblicato a Oxford, Moore, lo si sarà notato, adopera l'italiano, non l'inglese. Bei tempi! Del resto anche K. Witte aveva scritto in italiano i suoi Prolegomeni critici all'edizione della Commedia. Non c'era allora, a differenza di oggi, il pregiudizio anti-italiano, per cui qualche valutatore professionale dei tempi nostri sarebbe portato ad attribuire maggior punteggio a un saggio dantesco in inglese, solo perché, appunto, scritto in inglese. Ma torniamo alla parola dantofilo, che, nella pagina di Moore, equivale in sostanza a ciò che noi diremmo dantista.

\section{Il dantofilo di Carducci}

Siamo al ı 894, ma si può arretrare ancora. Infatti sarebbe stato strano che uno straniero, inglese, per quanto ottimo conoscitore della nostra lingua, inventasse parole nuove. Più verosimilmente, usava parole già esistenti.

Dantofilo, infatti, era stato usato nel I86I da un grande scrittore italiano, Giosue Carducci, recensendo, 
in maniera piuttosto critica ed ironica, su "La Nazione" di Firenze del 2r ottobre, un saggio di Buscaino Campo intitolato Il pié fermo di Dante. Cosi esordiva Carducci in quella recensione: "Ecco per certo genere di Dantofili un bocconcino ghiotto...". La recensione del i86I fu riedita nel i88I nelle Opere di Carducci, nel volume delle Ceneri e faville - Serie prima - 1959-1870 (pp. 317-I8). Per Carducci, la parola suonava piuttosto ironica, e nello stesso modo la usò anche altrove, per esempio nel passo riportato dal GDLI "Battaglia" che abbiamo già avuto modo di citare prima ${ }^{4}$. Così scriveva Carducci, polemicamente, nel I895, richiamando fra l'altro la precedente recensione del i86r:

se Dante potesse mai diventar noioso e dannoso, i dantisti o danteschi o dantofili avrebber finito con riuscire a farlo. E non intendo mica i dissertatori del su lodato piè fermo ${ }^{5}$ e gli spulciatori illustri delle varianti: la entomologia è in natura, e la filoleria ne ingrassa, e senza filoleria come si farebbe a spender quattrini per dar cattedre alla gente?[7]

Si sa, Carducci era piuttosto ruvido e spesso polemico (un po' come Dante, del resto), e aveva il gusto di certe parole difficili: qui, a dantofilo, si aggiunge filoleria, "erudizione eccessiva e pignola", una parola inventata sarcasticamente da Carducci, praticamente solo sua.

\section{Dantofilia senza confini}

La parola dantofilo ebbe fortuna, e circolò anche dopo essere stata usata dal Carducci, il quale (ricordiamolo) fu tra i fondatori nel s888 della Società Dantesca, e nel i889 tra i fondatori della Società Dante Alighieri. Dunque dantofilo ci riporta all'Ottocento, secolo chiave del culto di Dante. In quel periodo, Dante divenne per noi italiani una sorta di padre della patria, e le ricerche dantesche, sempre più raffinate, coinvolsero studiosi di altre nazioni, come il tedesco Witte e l'inglese Moore dei quali abbiamo parlato: perché Dante è nostro, ma è anche patrimonio della cultura europea e di tutta l'umanità.

Note:

I. Del resto Dantofilo è registrato nel Nóvo dizionario universale di P. Petrocchi, del I894 con la seguente definizione: "Chi à amore e fa raccolta delle òpere e delle edizioni di Dante". Insomma, una sorta di collezionista.

2. La data I879, però, resta riferimento oscuro. Si veda, più avanti, la nota 3 .

3. IL GDLI s.v. in prima posizione ha un rinvio a Carducci: "Se Dante potesse mai diventar noioso e dannoso, i dantisti o danteschi o dantofili avrebber finito con riuscire a farlo". La chiave: III-IO-429 rimanda all'edizione delle Opere, vol. Io, I950 e ss. Ovviamente si tratta di un'edizione moderna, alla quale non si poteva far riferimento per datare la parola. Per questo la datazione 1956 indicata dal GRADIT a stampa è un errore grossolano. La correzione nell'ed. GRADIT su chiavetta, però, resta oscura. Sicuramente non può riferirsi al passo citato dal GDLI "Battaglia", che è tratto dal saggio carducciano A proposito di un codice diplomatico dantesco, uscito sulla "Nuova antologia" del I5 agosto I895, e poi ripubblicato "con qualche emendazione" nel vol. delle Opere dedicato a Studi, saggi e discorsi, Bologna, Zanichelli, I898, pp. 355-374.

4. Cfr. supra, nota 3 .

5. Ovviamente si riferisce al titolo del libro di Buscaino Campo che aveva recensito nel i86I.

6. G. Carducci, Studi, Saggi e discorsi, Bologna, Zanichelli, I898, p. 363. 


\section{Cita come:}

Claudio Marazzini, Oggi siamo tutti dantofili: è il primo Dantedi della storia , "Italiano digitale", 2020, XII, 2020/1 (gennaio-marzo)

DOI: $10.35948 / 2532-9006 / 2020.3304$

Copyright 2020 Accademia della Crusca

Pubblicato con licenza creative commons CC BY-NC-ND 